\title{
In Situ Surface X-Ray Diffraction Studies of Homoepitaxial Electrochemical Growth on Au(100)
}

\author{
K. Krug, J. Stettner, and O. M. Magnussen \\ Institut für Experimentelle und Angewandte Physik, Universität Kiel, Olshausenstrasse 40, 24098 Kiel, Germany
}

(Received 17 January 2006; published 20 June 2006)

\begin{abstract}
Direct in situ $\mathrm{x}$-ray surface scattering studies of growth at a solid-liquid interface are demonstrated using the homoepitaxial electrodeposition on $\mathrm{Au}(100)$ as an example. With decreasing potential transitions from step-flow to layer-by-layer growth, manifested by layering oscillations in the x-ray intensity, then to multilayer growth, and finally back to layer-by-layer growth were observed. This complex growth behavior can be explained by the effect of anion coadsorbates and the potential-dependent Au surface reconstruction on the Au surface mobility.
\end{abstract}

PACS numbers: 81.15.Pq, 61.10.Nz, 68.08.-p, 68.55.Ac

The growth of crystalline solids in liquid solutions is central not only to natural mineralization but also to current and future technological deposition processes. To clarify the relationship between the atomic-scale structure of the solid-liquid interface, the growth behavior, and the resulting surface morphology, direct investigations of the interface structure during the growth process are required. In this work we demonstrate that this is possible by surface $\mathrm{x}$-ray scattering in transmission geometry up to growth rates of several monolayers per minute. An electrochemical system, homoepitaxial $\mathrm{Au}(100)$ electrodeposition, was chosen for this study because of the particularly easy control of electrochemical growth processes and the interesting potential-dependent growth behavior.

In contrast to crystal growth in solution, growth at the solid-vacuum interface has been investigated in great detail on the atomic scale for the case of molecular beam epitaxy (MBE) of metals and semiconductors by diffraction methods $[1,2]$. According to these experimental as well as theoretical studies [3] homoepitaxial growth far from equilibrium is governed by the flux of adatoms to the surface, i.e., the deposition rate, and the rates of intra- and interlayer transport, which are functions of temperature and surface structure. Dependent on the relative rates of these processes step-flow growth, layer-by-layer growth, or multilayer growth are observed. For (unreconstructed) fcc(100) surfaces step-flow or layer-by-layer growth is found over a wide range of temperatures, indicating highly effective interlayer mass transport [1]. For $\mathrm{Au}(100)$, which exhibits a reconstructed surface layer of hexagonally ("hex") arranged atoms under ultrahigh vacuum (UHV) conditions, also layer-by-layer growth with strongly anisotropic islands was reported $[4,5]$.

In the present in situ surface x-ray scattering (SXS) study of electrochemical homoepitaxial growth on $\mathrm{Au}(100)$ we aim at elucidating the influence of the deposition parameters and interface structure on the atomistic growth process and on the resulting atomic-scale film morphology in a similar manner as in the previous MBE studies. In electrochemical environment the hex surface reconstruction is stable only negative of a critical (electrolyte-dependent) potential, whereas at more positive potentials the surface is unreconstructed $[6,7]$. Furthermore, the surface mobility in the potential regime of the unreconstructed $\mathrm{Au}(100)$ surface was found to increase substantially with increasing potential, in particular, in the presence of $\mathrm{Cl}^{-}$anions [811]. According to kinetic growth theory also the growth behavior should therefore depend on potential, as will indeed be shown in this work. Deposition was performed at high overpotentials, where the deposition rate is determined solely by diffusion of the metal species $\left(\mathrm{AuCl}_{4}{ }^{-}\right)$in the electrolyte solution to the metal surface, i.e., can be controlled by the metal concentration in the solution. This procedure allows us to independently control the potential and the deposition rate over a wide range of potentials and growth rates, making it possible to separate the influence of these two effects on the atomic-scale morphology of the deposit [12].

In situ SXS measurements were performed at beam line ID32 of the ESRF and beam line BW2 of HASYLAB using photon energies of 18.2 and $10.0 \mathrm{keV}$, respectively. For the SXS experiments a $1 \mathrm{ml}$ electrolyte holding hanging meniscus cell with a Pt counter electrode and a $\mathrm{Ag} / \mathrm{AgCl}(3 \mathrm{M}$ $\mathrm{KCl}$ ) reference electrode was used, where the $\mathrm{x}$-ray beam passes through a $50 \mu \mathrm{l}$ freestanding electrolyte meniscus of nearly cylindrical shape, which is surrounded by an $\mathrm{N}_{2}$ atmosphere to keep the electrolyte oxygen free [12]. This cell allows parallel high-quality electrochemical measurements and in situ SXS studies of diffusion controlled deposition as well as electrolyte exchange during the diffraction experiments by a remote-controlled pump system. The $\mathrm{Au}(100)$ single crystal sample (Mateck, miscut $<$ $0.1^{\circ}$, mosaic spread $<0.2^{\circ}$ ) was prepared prior to the experiments by flame annealing. As electrolyte $0.1 \mathrm{M} \mathrm{HCl}+$ $x \mathrm{mM} \mathrm{HAuCl}_{4}(x=0.05,0.1,0.2,0.5)$ solutions, prepared from suprapure $\mathrm{HCl}$ (Merck), $\mathrm{HAuCl}_{4}$ (Johnson Matthey), and Milli- $Q$ water, were employed, corresponding to diffusion-limited deposition rates of approximately 0.4 to $4 \mathrm{ML} / \mathrm{min}$ [13]. In each experiment the sample was first immersed in $\mathrm{HAuCl}_{4}$-free $0.1 \mathrm{M} \mathrm{HCl}$ for alignment and first SXS characterization to ensure a well-ordered hex reconstructed $\mathrm{Au}$ surface prior to deposition. Subse- 
quently, the electrolyte was exchanged by Au-containing solution, resulting in the onset of electrodeposition.

To study the growth behavior the scattered intensity was monitored as a function of time at selected reciprocal space positions (typically close to the anti-Bragg positions) along the specular $(0,0)$ and nonspecular $(1,1)$ crystal truncation rods (CTR). In situ studies of the growth mechanism were performed in $\mathrm{HAuCl}_{4}$-containing solution using the following procedure: first, the potential was kept for at least $5 \mathrm{~min}$ at $0.6 \mathrm{~V}$, where the Au surface mobility is very high (see below), resulting in rapid smoothening even of very rough surfaces as verified by the complete recovery of the $\mathrm{x}$-ray intensity. Then the Au concentration was replenished by exchange with fresh electrolyte solution and after a waiting time of at least 4 min a potential step to a more negative potential was initiated. Because of the change in surface mobility induced by the potential step, significant changes in the scattered intensity are observed, from which the kinetic growth mode can be inferred. Examples of the scattered intensity as a function of time after the potential step are shown in Fig. 1 for electrolytes containing different $\mathrm{HAuCl}_{4}$ concentrations. At all employed concentrations oscillations in the $\mathrm{x}$-ray intensity are observed that clearly indicate layer-by-layer growth, with each oscillation period $\Delta t$ corresponding to the deposition of one $\mathrm{Au}$ monolayer [1,2]. These growth oscillations are found in the specular [Figs. 1(b) and 1(c)] as well as the nonspecular [Figs. 1(a) and 1(d)] CTRs and occur in a wide potential regime. The amplitude of these oscillations decays within typically 3 to 4 periods, approaching an approximately constant value. Overall, this behavior strongly resembles that found in various scattering studies by He scattering, electron diffraction (RHEED, MEED, and LEED), and SXS of

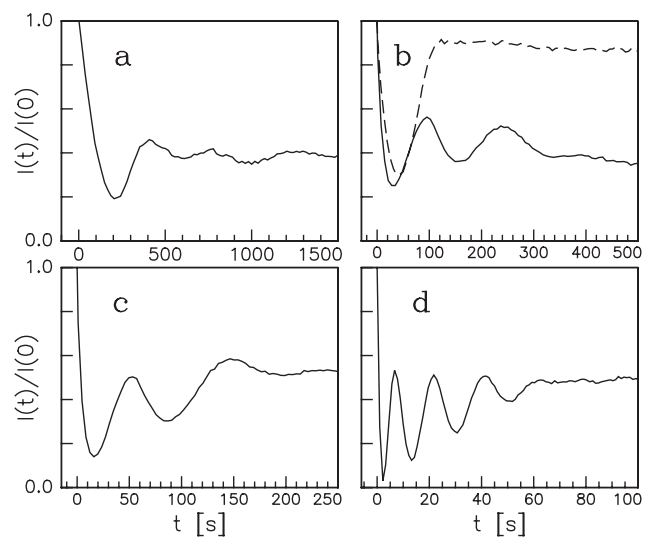

FIG. 1. In situ growth experiments on $\mathrm{Au}(100)$ in $0.1 \mathrm{M} \mathrm{HCl}$ solution containing (a) $0.05 \mathrm{mM}$, (b) $0.1 \mathrm{mM}$, (c) $0.2 \mathrm{mM}$, and (d) $0.5 \mathrm{mM} \mathrm{HAuCl} \mathrm{H}_{4}$ at a deposition potential of (a) $0 \mathrm{~V}$, (b) $0.05 \mathrm{~V}$ (solid line) and $0.2 \mathrm{~V}$ (dashed line), (c) $0.10 \mathrm{~V}$, and (d) $0.25 \mathrm{~V}$. The panels show the background-corrected x-ray intensity $I(t)$ as a function of deposition time $t$, normalized with respect to the saturation value $I(0)$ directly before the potential step at $t=0$. The data were obtained at reciprocal space positions of (a),(d) $(1,1,0.1)$ and (b),(c) $(0,0,0.7)$; similar behavior was found at other positions along the CTRs.
MBE growth on (100)-oriented fcc-metal substrates under UHV conditions [1,2]. Similar oscillations could be observed at all $\mathrm{HAuCl}_{4}$ concentrations employed in this study, albeit in somewhat different potential regimes.

As visible in Fig. 1, the oscillation period $\Delta t$ decreases, i.e., the local deposition rate increases, approximately proportionally to the concentration, as expected for diffusionlimited deposition [14]. Within the variation of the data the $\Delta t$ values are in good agreement with the deposition rates, obtained from the electrochemical current density. The decay in the oscillation amplitude with time can be attributed to slow surface roughening due to nonideal layer-bylayer growth. Similar behavior was also found for MBE under UHV conditions. Following the procedure commonly employed in MBE studies [1] the presence of at least one complete period in the oscillations will be associated with layer-by-layer growth in the following.

The response of the scattered intensity $I(t)$, especially the occurrence of growth oscillations, strongly depends on potential. At the most positive potentials $(>0.4$ and $>0.1 \mathrm{~V}$ in $0.1 \mathrm{M} \mathrm{HCl}$ containing 0.1 and $0.5 \mathrm{mM} \mathrm{HAuCl}_{4}$, respectively) the intensity usually first decreases and then recovers, approaching an approximately constant intensity that is comparable to the initial intensity $I_{0}$ before the potential step [Fig. 1(b), dashed line]. The steady-state intensity is obtained precisely after the time $\Delta t$, corresponding to the deposition of the first monolayer. This behavior is attributed to Au island nucleation and subsequent $2 \mathrm{D}$ growth (i.e., a layer-by-layer growth) of the first monolayer, followed by step-flow growth (see below). After stepping the potential down to more negative values growth oscillations emerge and, at even more negative potentials, disappear again. This is illustrated in Fig. 2
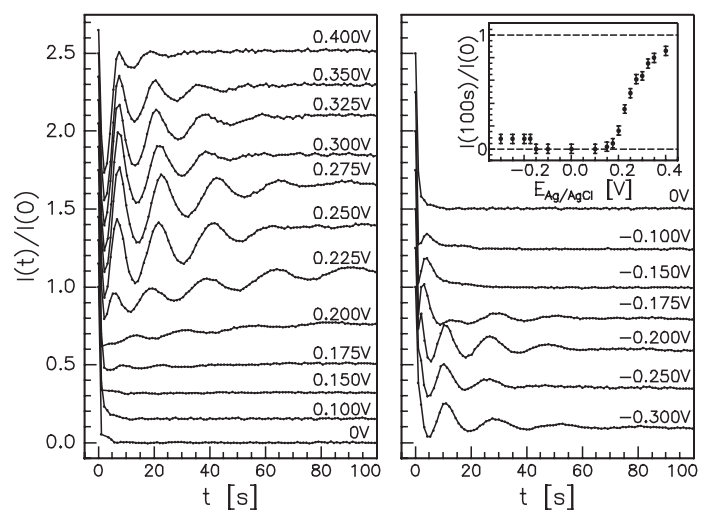

FIG. 2. Background-subtracted, normalized intensity as a function of deposition time $t$ after potential steps from $0.6 \mathrm{~V}$ to different potentials in $0.1 \mathrm{M} \mathrm{HCl}+0.5 \mathrm{mM} \mathrm{HAuCl}$. For clarity, the curves are shifted with respect to each other by a constant offset. The data were obtained at $(1,1,0.1)$ and show step-flow, layer-by-layer, multilayer, and reentrant layer-bylayer growth (for classification of each curve, see Fig. 3, topmost row of symbols) with decreasing potential. In the inset the steady-state intensity, measured $100 \mathrm{~s}$ after the potential step, is plotted as a function of potential. 
for a series of measurements in $0.1 \mathrm{M} \mathrm{HCl}+0.5 \mathrm{mM}$ $\mathrm{HAuCl}_{4}$ solution, where the oscillations are found between approximately 0.40 and $0.20 \mathrm{~V}$. Below $0.20 \mathrm{~V} I(t)$ decays rapidly to zero (see Fig. 2, inset), indicating formation of a rough surface via multilayer growth. Interestingly, upon further lowering of the potential growth oscillations reappear negative of $-0.15 \mathrm{~V}$ (Fig. 2, right panel). As verified by separate studies of the $\mathrm{Au}(100)$ in-plane structure, performed in the same in situ SXS experiments, the onset of this "reentrant" layer-by-layer growth coincides with the formation of the hex surface reconstruction on the electrode surface. Analog experiments at deposition rates of $\approx 0.38$ and $\approx 0.63 \mathrm{ML} / \mathrm{min}$ (not shown) revealed a similar behavior. However, here the transition from stepflow to layer-by-layer growth is found at more negative potentials $(<0.20$ and $<0.15 \mathrm{~V}$ at fluxes of $\approx 0.63$ and $\approx 0.38 \mathrm{ML} / \mathrm{min}$, respectively) and the multilayer growth regime is limited to a small potential regime around $0 \mathrm{~V}$ at $\approx 0.63 \mathrm{ML} / \mathrm{min}$ whereas it does not occur at all at $\approx 0.38 \mathrm{ML} / \mathrm{min}$. These observations, which are summarized in the kinetic growth mode diagram in Fig. 3, reveal that the growth process and the resulting morphology depend in a complex way on the deposition potential as well as deposition rate.

Since the flux is independent of potential in these experiments, the potential-dependent growth behavior has to be related to a potential dependence of the surface transport processes. In the potential regime of the unreconstructed $\mathrm{Au}(100)$ surface $(\geq-0.15 \mathrm{~V}$ in $0.1 \mathrm{M} \mathrm{HCl}+0.5 \mathrm{mM}$ $\mathrm{HAuCl}_{4}$ solution) a change from multilayer to layer-bylayer, and then to step-flow growth is found with increasing potential, indicating a continuous increase in the surface mobility. The data are in complete agreement with previous studies of surface transport in Au-free solution, where a substantial, often exponential increase in surface mobility with increasing potential was reported [8-11]. This potential effect was attributed to the influence of the electric field of the electrochemical double layer and the

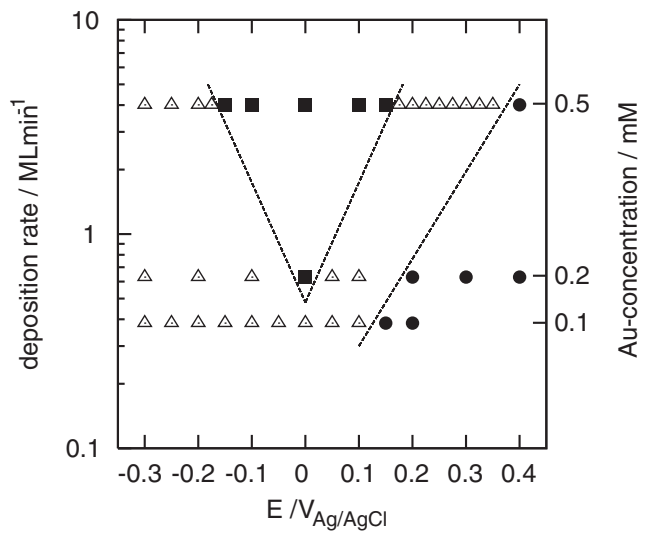

FIG. 3. Kinetic growth mode diagram for $\mathrm{Au} / \mathrm{Au}(100)$ in $0.1 \mathrm{M} \mathrm{HCl}$, showing the occurrence of step-flow (filled circles), layer-by-layer (open triangles), and multilayer growth (filled squares) as a function of potential and deposition rate. chemisorption of anions on the activation energies for surface transport [11]. Specifically, the presence of $\mathrm{Cl}^{-}$ anions is known to significantly enhance the surface mobility $[8,10,15]$.

Within the framework of kinetic growth theory an increase in the surface transport rate at identical adatom flux $F$ should result in a crossover from multilayer to layer-bylayer to step-flow growth, as indeed found in the experiments in solution containing $0.5 \mathrm{mM} \mathrm{HAuCl}$. The potential-dependent 3D-2D growth transition indicates an increased rate of interlayer transport or, more precisely, a decrease in the Ehrlich-Schwoebel barrier $E_{S}$ for Au adatoms stepping down the Au islands. Support for this comes from in situ AFM experiments on the decay of multilayer islands on $\mathrm{Au}(100)$ in $\mathrm{Cl}^{-}$-containing electrolyte, where a pronounced increase in the island decay rate with potential was found [10], indicating likewise enhanced interlayer transport. With decreasing deposition rate the potential regime of multilayer growth is reduced. This again is in accordance with the condition for the 2D-3D transition and can be rationalized by the lower nucleation probability for $\mathrm{Au}$ islands at lower flux, resulting in layer-by-layer growth even at lower surface transport rates. More quantitatively, according to rate equation theory and kinetic Monte Carlo simulations the 2D-3D transition line in the growth mode diagram is given (for a critical nucleus size $i=1$, an adatom diffusion rate on the terraces $D$, and an in-plane lattice constant $a$ ) by the condition $\exp \left(-\Delta E_{S} / k_{B} T\right) \propto$ $\left(D / F a^{4}\right)^{-1 / 6}[16-18]$, where $E_{D}$ is the activation energy for surface diffusion and $\Delta E_{S}=E_{S}-E_{D}$ the additional barrier for step-down diffusion. At constant $T$ this condition can be rewritten as $\frac{5}{6} E_{D}-E_{S} \propto \ln F$, i.e., the critical flux $F$ for the transition from 2D to 3D growth only shifts to higher values, if the Ehrlich-Schwoebel barrier $E_{S}$ decreases whereas a decrease in the surface diffusion rate $E_{D}$ (at constant $E_{S}$ ) would even lower $F$. Hence, the observed increase in $F$ towards more positive potentials (Fig. 3) suggests that with increasing potential the EhrlichSchwoebel barrier decreases by (at least) a similar amount as the surface diffusion barrier.

The 2D to step-flow growth transition depends on the average step separation, i.e., the surface morphology, and hence is of less fundamental importance. Step flow emerges when intralayer transport to existing steps is so rapid that all adatoms reach the steps prior to nucleation and consequently depends on the diffusion barrier for adatom motion on the terraces, the binding energy of the critical cluster, and the adatom flux [1]. Taking into account the increase in intralayer transport with increasing potential, the transition to step flow at positive potentials and the dependence of this transition on the deposition rate can be easily rationalized.

The reentrant layer-by-layer growth in the potential regime of the hex reconstructed surface is in good agreement with the homoepitaxial growth behavior of reconstructed $\mathrm{Au}(100)$ under UHV conditions, where at similar 
deposition rates also a layer-by-layer growth was found $[4,5]$. It indicates an enhanced surface mobility in the reconstructed as compared to the unreconstructed surface. Indeed theoretical studies predict considerably lower barriers for adatom surface diffusion on the hexagonal closepacked reconstructed $\mathrm{Au}(100)$ surface [19], but no data on the additional barrier at step edges $\Delta E_{S}$ exists, which determines the interlayer transport and consequently the 3D-2D transition. Our experiments suggest that the formation of the hex reconstruction substantially lowers this barrier, in accordance with in situ AFM observations [9]. However, the atomic-scale growth mechanisms on the reconstructed $\mathrm{Au}(100)$ surface are rather complex and cannot be rationalized based on the simple kinetic models employed above: first, the critical nucleus size $i>1$ on reconstructed $\mathrm{Au}(100)$ [4] and consequently the simple relationship for the 2D-3D transition given in Refs. [1618] does not hold anymore. Second, molecular dynamics simulations suggest that rather complex collective processes are involved in island nucleation [20]. Third, recent STM studies revealed other, more complex mass transport mechanisms on partly reconstructed $\mathrm{Au}(100)$, such as fast adatom transport along the boundaries between reconstructed and unreconstructed surface areas and the quasicollective motion of elements of the surface reconstruction $[19,21]$. More complex models will therefore be necessary to describe growth on the hex reconstructed surface.

Although the experiments discussed here resemble diffraction studies of MBE growth under vacuum conditions, some important differences to those studies exist. In both cases the high scattered intensity indicates that the initial surface is smooth with large atomically flat terraces. However, whereas conventional MBE growth studies monitor the surface morphology at fixed temperature (i.e., fixed surface mobility) after initiating a constant adatom flux, our experiments show the response of the surface morphology at constant (diffusion-limited) flux upon a potential step, i.e., upon a change in surface mobility. This results in an enhanced transient adatom concentration on the Au terraces directly after the potential step until a new equilibrium adatom distribution has developed. The layer-by-layer growth of the first monolayer in the step-flow potential regime may be attributed to this phenomenon. An alternative experimental approach would be electrodeposition experiments that, as in MBE, start at zero flux. This can in principle be done by rapid exchange of Au-free with Au-containing solution at fixed potential.

In summary, our experiments demonstrate that by surface $\mathrm{x}$-ray scattering in transmission geometry direct in situ studies of the kinetic growth mode are possible not only for MBE, but also for growth at solid-liquid interfaces. As shown here for homoepitaxial electrodeposition on $\mathrm{Au}(100)$ both the solid surface structure, e.g., the $\mathrm{Au}$ reconstruction, as well as the solution side of the interface, such as coadsorbed species, can affect the surface transport of the deposited adatoms and by this the growth behavior. Similar studies may help to clarify other fundamental as well as applied problems, e.g., the role of organic additives in electrodeposition or biomineralization processes.

We gratefully acknowledge beam time by the ESRF and HASYLAB, help at the ID32 beam line and technical support by F. Renner, T.-L. Lee, and J. Zegenhagen, technical support at BW2 by H. Schulte-Schrepping and W. Drube, and financial support by the Deutsche Forschungsgemeinschaft (MA 1618/13-1).

[1] G. Rosenfeld, B. Poelsema, and G. Comsa, in Growth and Properties of Ultrathin Epitaxial Layers, edited by D. A. King and D.P. Woodruff (Elsevier Science, Amsterdam, 1999), Vol. 8, p. 66.

[2] M. V. R. Murty, T. Curcic, A. Judy, B. H. Cooper, A. R. Woll, J. D. Brock, S. Kycia, and R. L. Headrick, Phys. Rev. B 60, 16956 (1999).

[3] J. A. Venables, G. D. T. Spiller, and M. Hanbrücken, Rep. Prog. Phys. 47, 399 (1984).

[4] S. Günther, E. Kopatzki, M. C. Bartelt, J. W. Evans, and R. J. Behm, Phys. Rev. Lett. 73, 553 (1994).

[5] S. Günther, Ph.D. thesis, Universität Ulm, 1995.

[6] D. M. Kolb, Prog. Surf. Sci. 51, 109 (1996).

[7] B. M. Ocko, J. Wang, A. J. Davenport, and H.S. Isaacs, Phys. Rev. Lett. 65, 1466 (1990).

[8] R. J. Nichols, O. M. Magnussen, J. Hotlos, T. Twomey, R. J. Behm, and D. M. Kolb, J. Electroanal. Chem. 290, 21 (1990).

[9] N. Ikemiya, M. Nishide, and S. Hara, Surf. Sci. 340, L965 (1995).

[10] K. Kubo, N. Hirai, and S. Hara, Appl. Surf. Sci. 237, 301 (2004).

[11] M. Giesen, G. Beltramo, S. Dieluweit, J. Müller, H. Ibach, and W. Schmickler, Surf. Sci. 595, 127 (2005).

[12] A. H. Ayyad, J. Stettner, and O. M. Magnussen, Phys. Rev. Lett. 94, 066106 (2005).

[13] All given growth rates in this Letter were determined from the time between the first and the second maximum in the $I(t)$ curves.

[14] The reproducibility of the oscillation periods is limited by (a) the rather slow approach of steady-state hydrodynamic conditions after the exchange (3-5 min), which is also the origin of the slow increase in $\Delta t$ observed in some of the experiments, and (b) the slow depletion of the Au concentration within the electrolyte, resulting in a $1 \%$ decrease in deposition rate per minute.

[15] D. J. Trevor, Ch. E. D. Chidsey, and S. N. Loiacono, Phys. Rev. Lett. 62, 929 (1989).

[16] J. Tersoff, A. W. Denier van der Gon, and R. M. Tromp, Phys. Rev. Lett. 72, 266 (1994).

[17] J. Rottler and P. Maass, Phys. Rev. Lett. 83, 3490 (1999).

[18] J. Krug, P. Politi, and T. Michely, Phys. Rev. B 61, 14037 (2000).

[19] M. Labayen, C. Ramirez, W. Schattke, and O.M. Magnussen, Nat. Mater. 2, 783 (2003)

[20] M. Nomura and X.-Q. Wang, Phys. Rev. Lett. 81, 2739 (1998).

[21] M. Labayen and O. M. Magnussen, Surf. Sci. 573, 128 (2004). 